\title{
Catalytic Asymmetric Synthesis of Allylic Aryl Ethers
}

Stefan F. Kirsch, Larry E. Overman, Nicole S. White

Department of Chemistry, 516 Rowland Hall, University of California, Irvine, CA 92697-2025

\section{Supporting Information}

(Total Pages: 136)

$\underline{\text { Index }}$

$\underline{\text { Page }}$

General Experimental Details

S2

Characterization Data for Trichloroacetimidates

S3

General Procedure for the Enantioselective Allylic Etherification

S4

Catayzed by $[\mathrm{COP}-\mathrm{OAc}]_{2}$

Characterization Data for Allylic Aryl Ethers

S4

${ }^{1} \mathrm{H}$ and ${ }^{13} \mathrm{C}$ NMR spectra of trichloroacetimidates and allyl aryl ethers

$\mathrm{S} 14$

HPLC or SFC traces used to determine enantiopurity

Experimental Details for Methods Employed to Establish Absolute

S108

Configuration of Allyl Aryl Ether Products

Characterization Data for Samples Used to Determine Absolute Configuration

${ }^{1} \mathrm{H}$ and ${ }^{13} \mathrm{C}$ NMR spectra of new 3-aryloxy-1-alkynyl intermediates

S112

HPLC or SFC traces used to determine absolute configuration

S127 

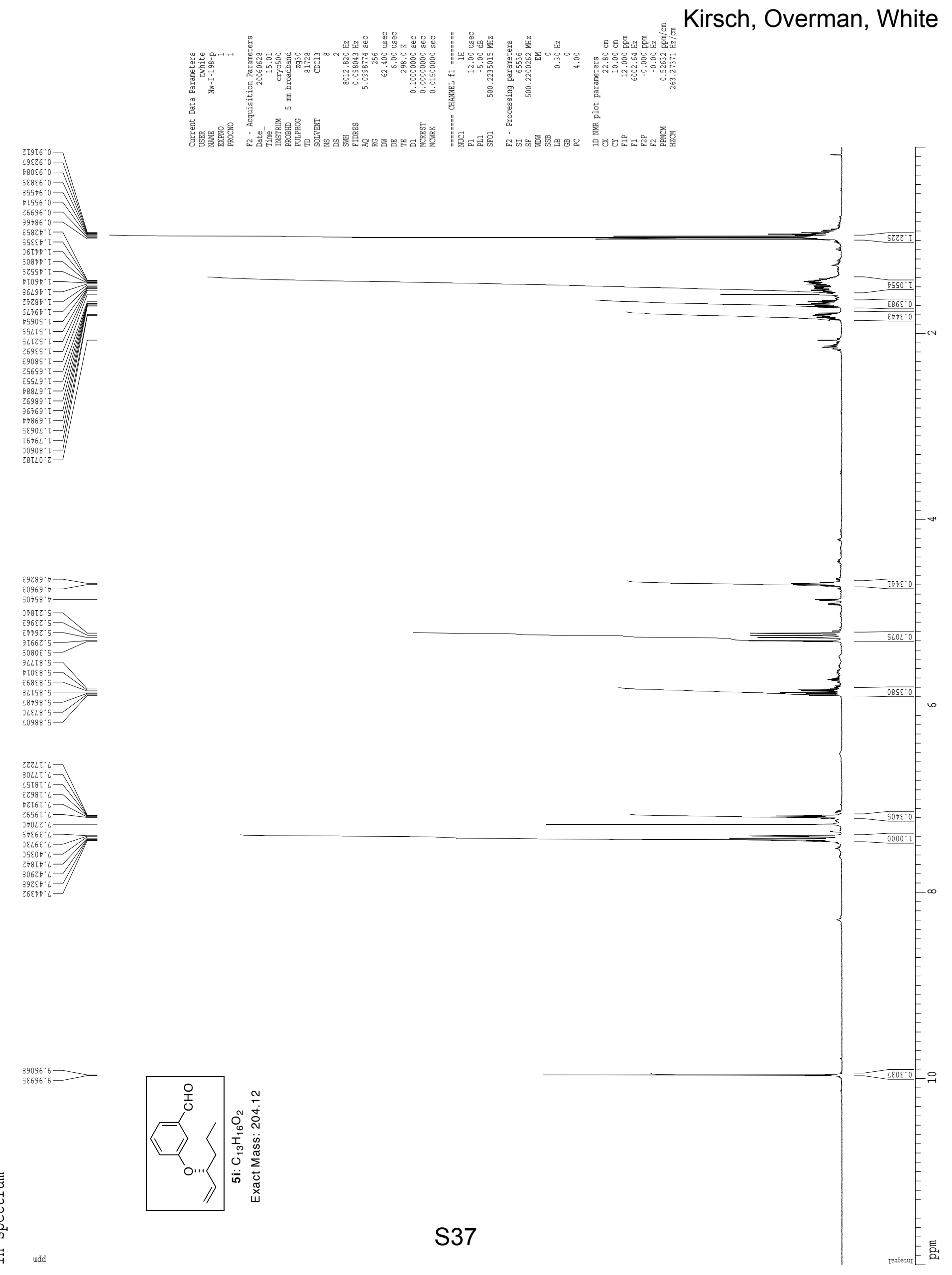

Kirsch, Overman, White 
Kirsch, ${ }_{5}$ Overman, White
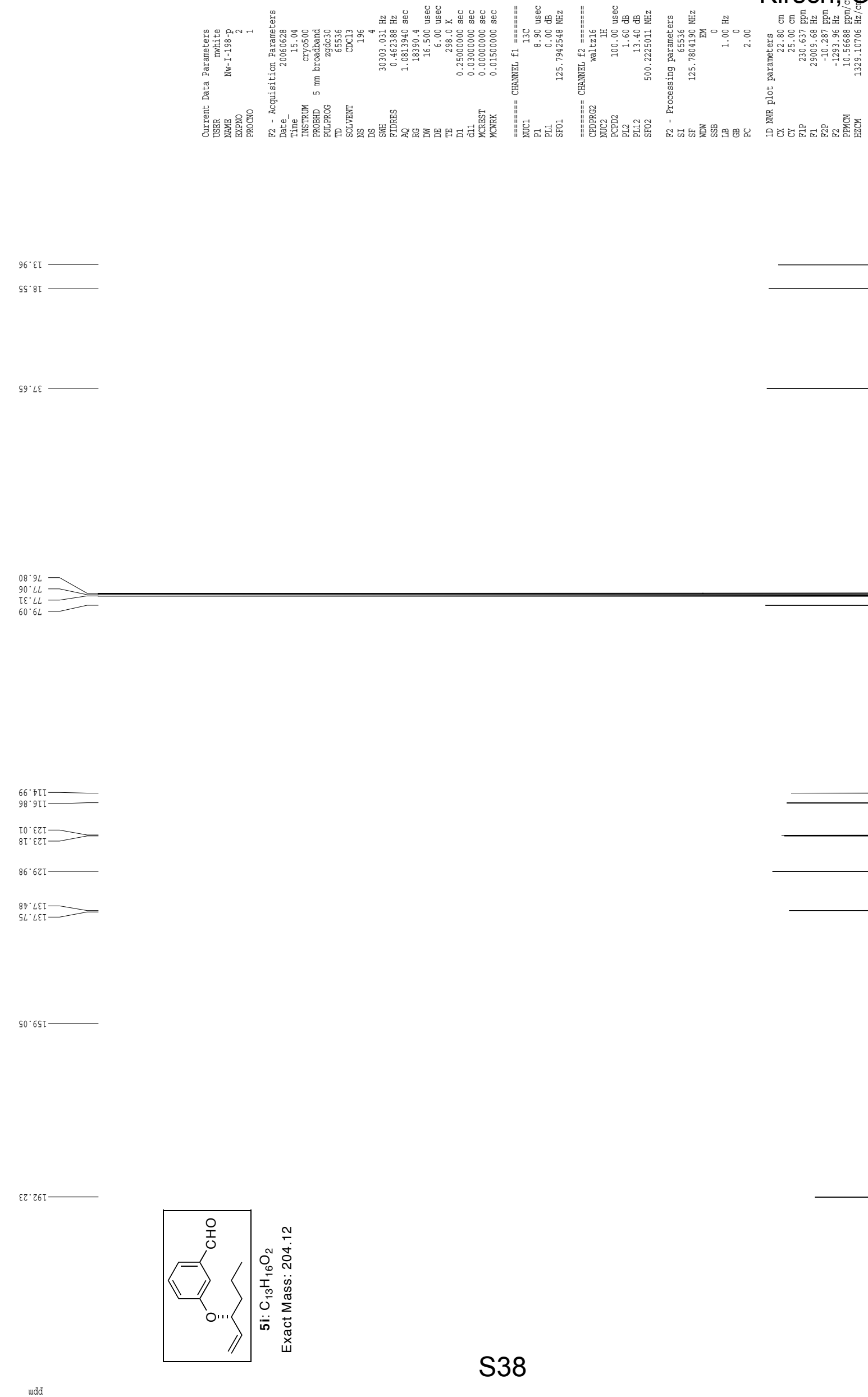
Kirsch, Overman, White
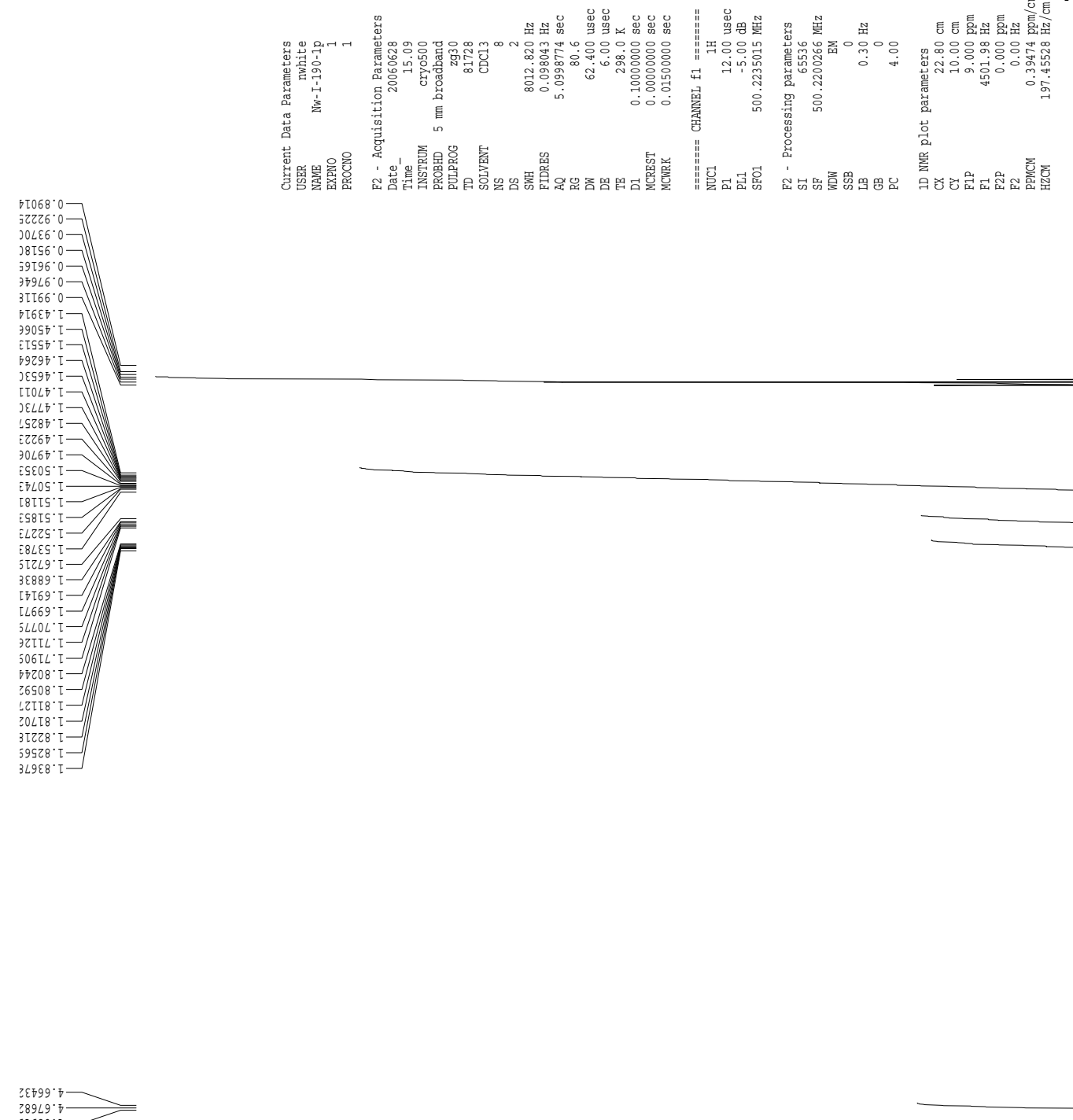

$289 \angle 9^{\circ} 7$

(2)

ZLTSR'S

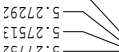

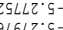

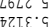

गЋZIE'S

iโ $208 \cdot \mathrm{s}$

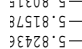

उЕ६Z8.

39098.9

ET698. 9
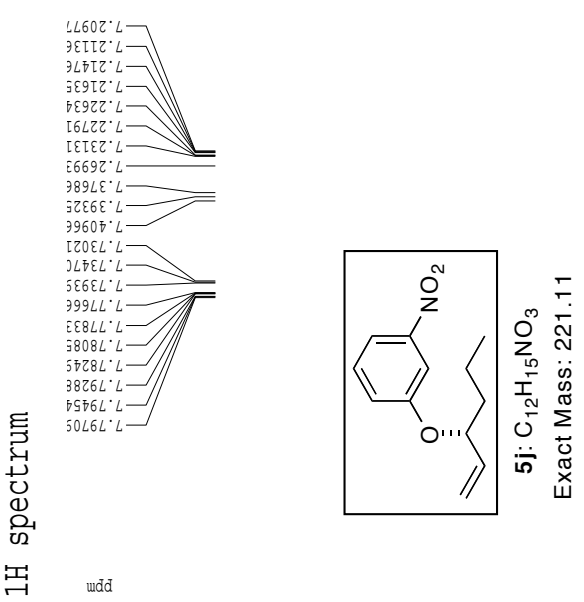

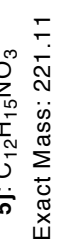


Kirsch, ${ }_{5}$ Overman, White
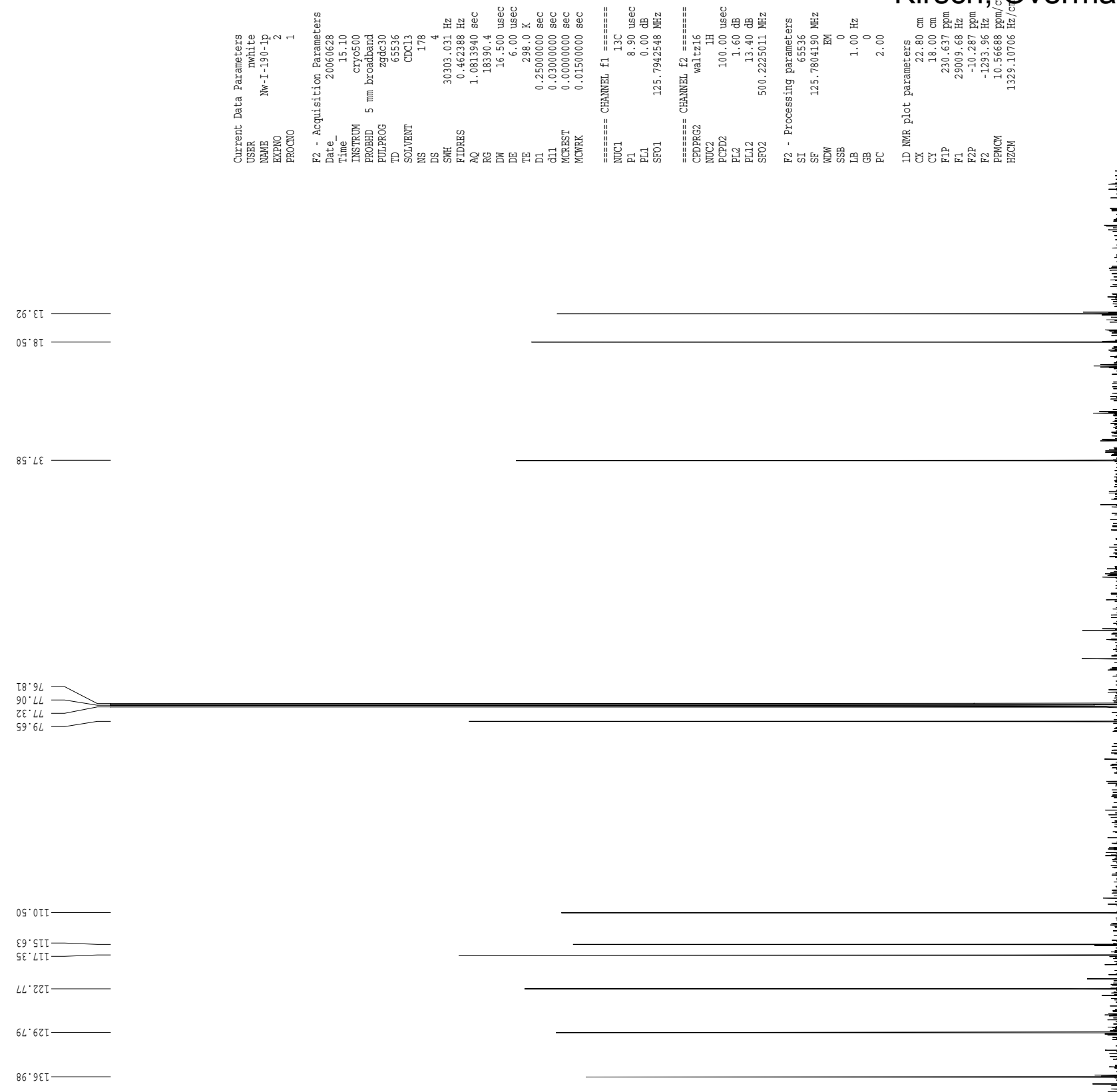

$9 \tau \cdot 6 \pi \tau$
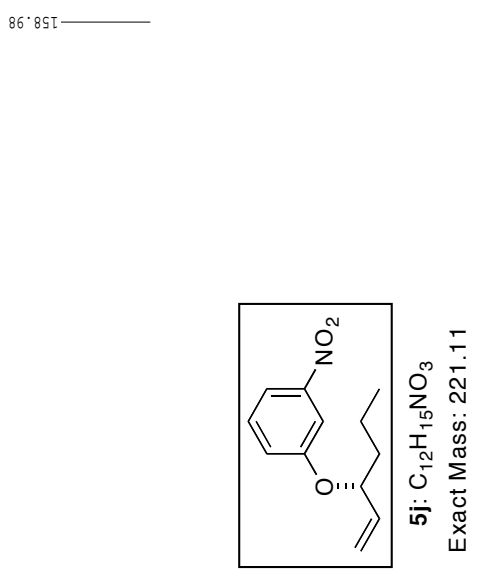


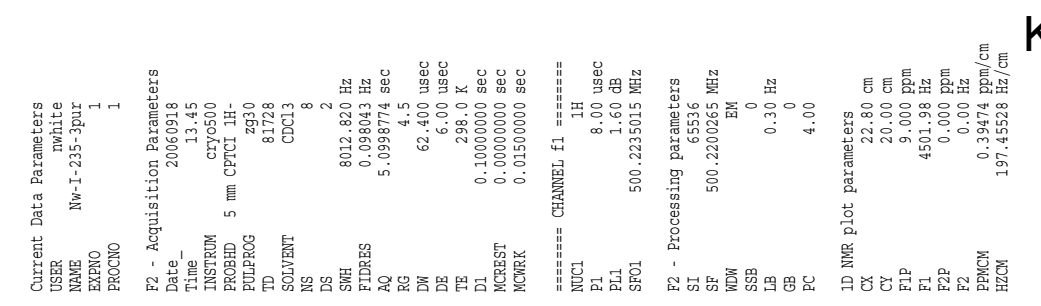

Kirsch, Overman, White

โL8E0. $T$

$32870^{\circ} \mathrm{T}$
$\angle 8990^{\circ} \mathrm{T}$

EहE80 $\mathrm{L}$

วLฑ

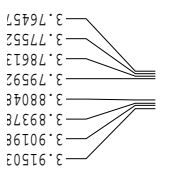

${ }^{30929^{\circ} 7-}$

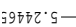

$2.2699 \cdot \cdot 9$

jELIE. 5

$30098^{\circ} \mathrm{g}$

$92768 \cdot 5$

รโ $988 L \cdot 9$

$5 \angle 66 L \cdot 9$

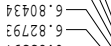

$46 \varepsilon \varepsilon 8.97$

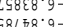

эTZS 8.9

$56692^{\circ} \mathrm{L}$

उLL9E.

โโ $96 \varepsilon^{\circ}: L$

${ }^{J \operatorname{IIIT} \cdot L} \cdot L$

$38627 \cdot L$

$26 L \varepsilon \mp: L-$

LLTST:LC

ถZ9ST:L二

it879 0

$3 \mathrm{~F}^{2} 99^{\circ} \mathrm{L}-$

$55 \angle 89^{\circ} \mathrm{L}-$

$10689^{\circ} \mathrm{L}-$

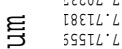

总 $599 L 2 \cdot L=$

ญ्,

舀 wadd
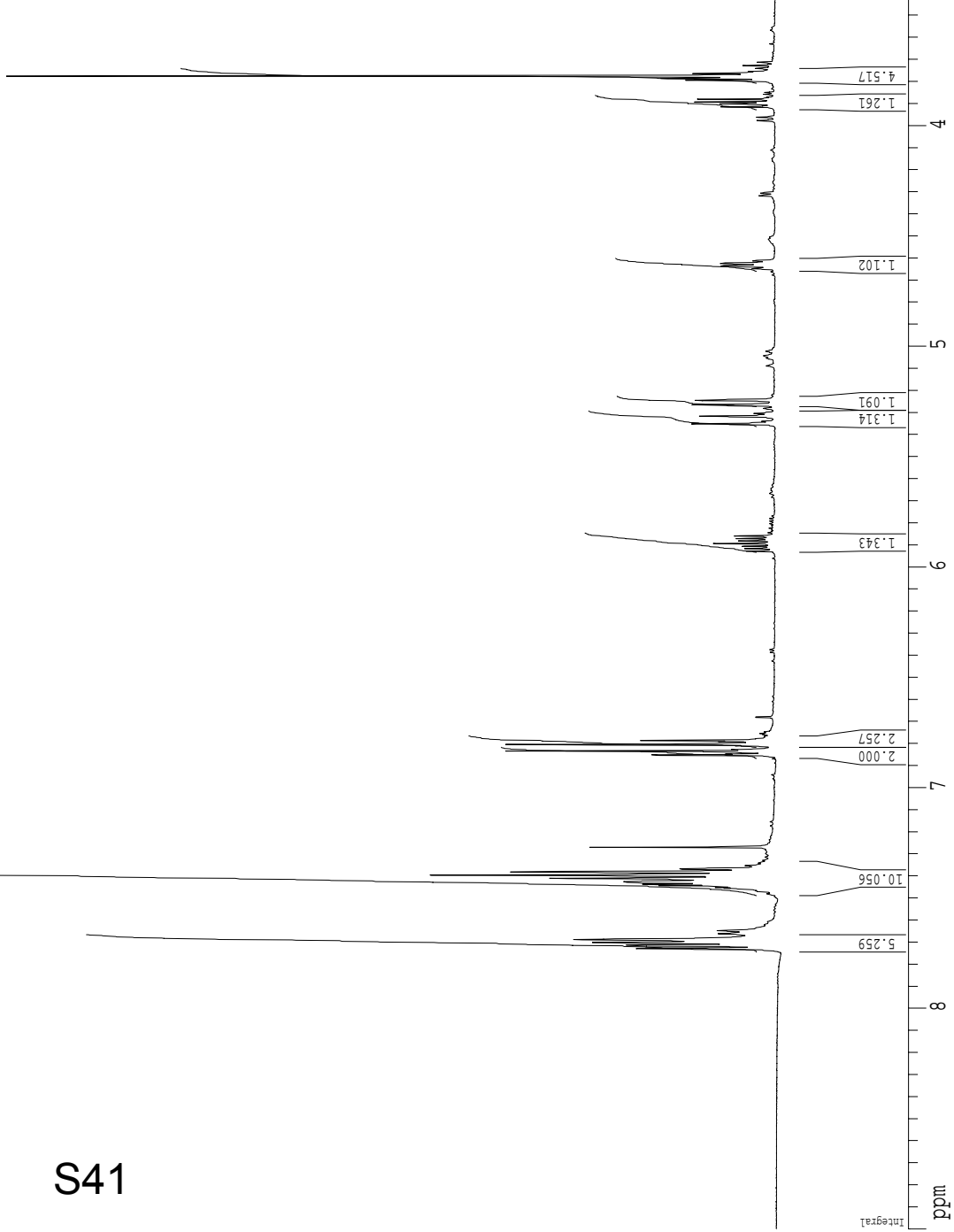
Kirsch, ${ }_{5}$ Overman, White
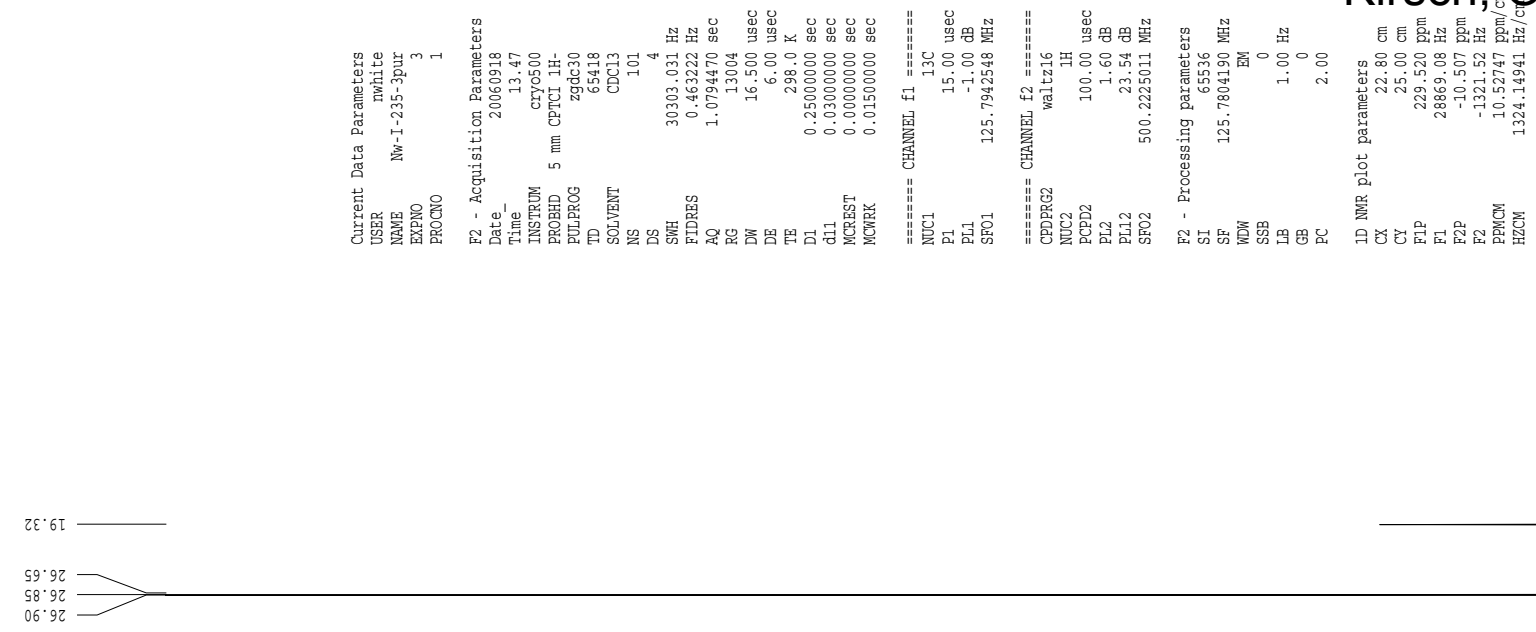

ऽ. ५ -

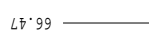

$\varepsilon 8^{\circ} \cdot 9 \mathrm{~L}$

$80^{\circ} L L$
$7 \varepsilon \cdot L L$
$08^{\circ} 08$
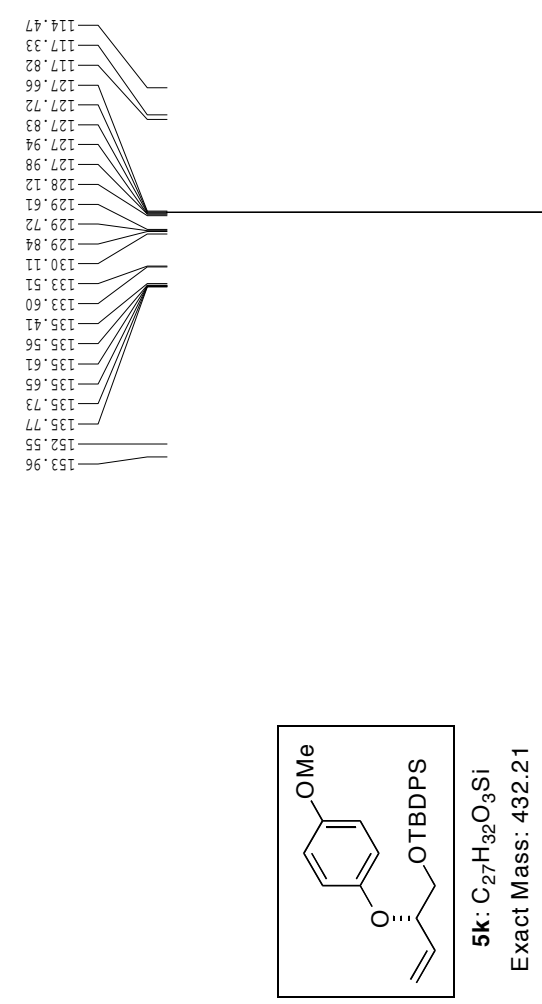
Kirsch, Overman, White
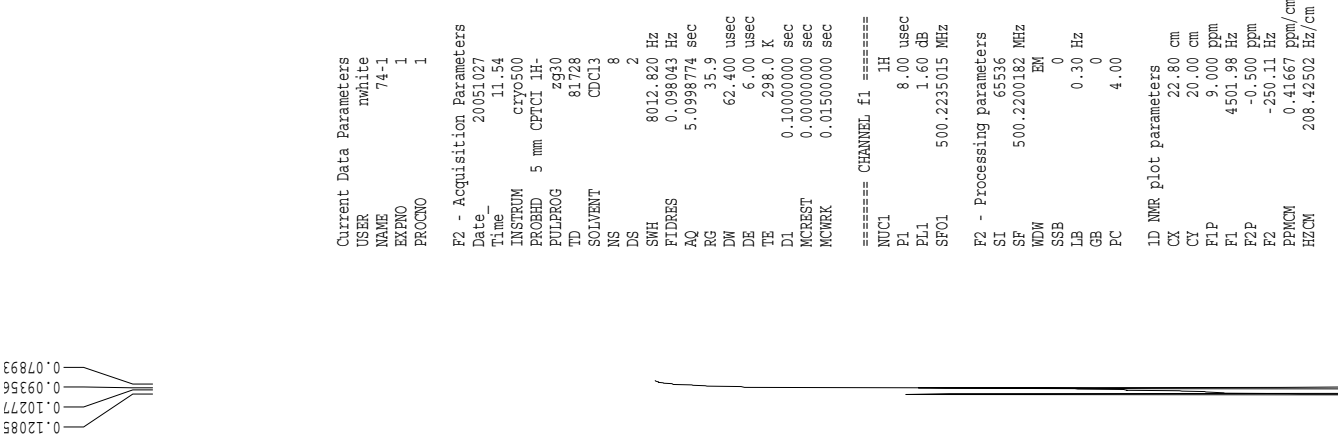

ธع66L 0

${ }_{90 \varepsilon \mp 6 \cdot 0}^{5 T 616}$

$76 \varepsilon 9 L \cdot \varepsilon-$
$0 L \varepsilon L L \cdot \varepsilon-1$

$0 L E L L \cdot \varepsilon-1$
$\sigma \varepsilon S 8 L \cdot \varepsilon-1$

ZTS6L $L$. $\varepsilon$

$0 \mp T 98^{\circ} \varepsilon$
$\varepsilon 97 \angle 8^{\circ} \varepsilon$

$\angle L Z 88^{\circ} \varepsilon$

${ }^{0} 0968 \cdot \varepsilon$

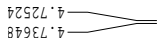

$80 \operatorname{Tog}^{\circ} \mathrm{c}$

$5 T 69 \varepsilon^{\circ} \cdot 9-$

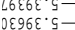

89606.5

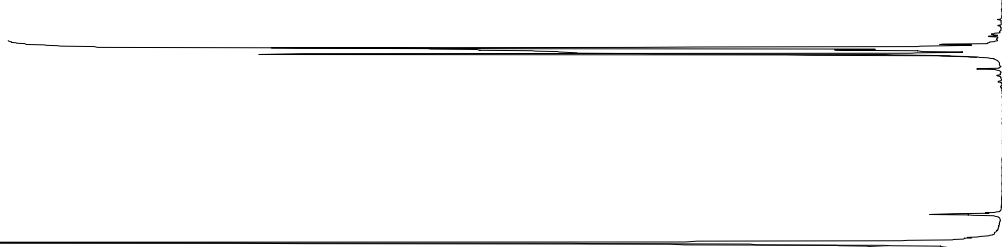

3
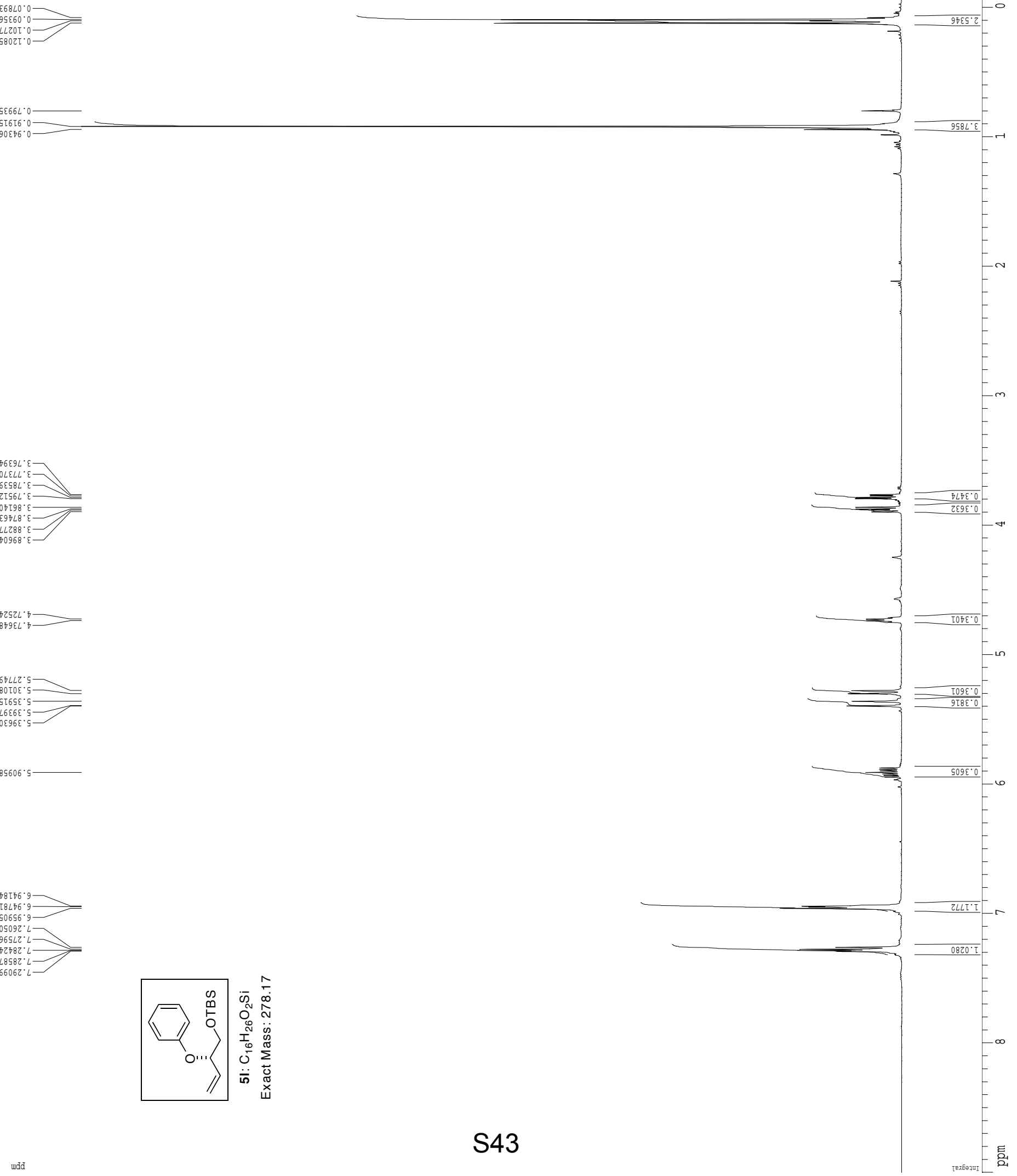
Kirsch, ${ }_{5}$ Overman, White

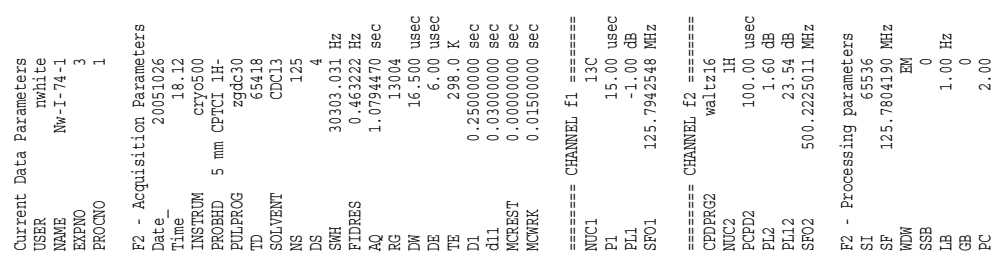

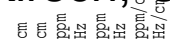
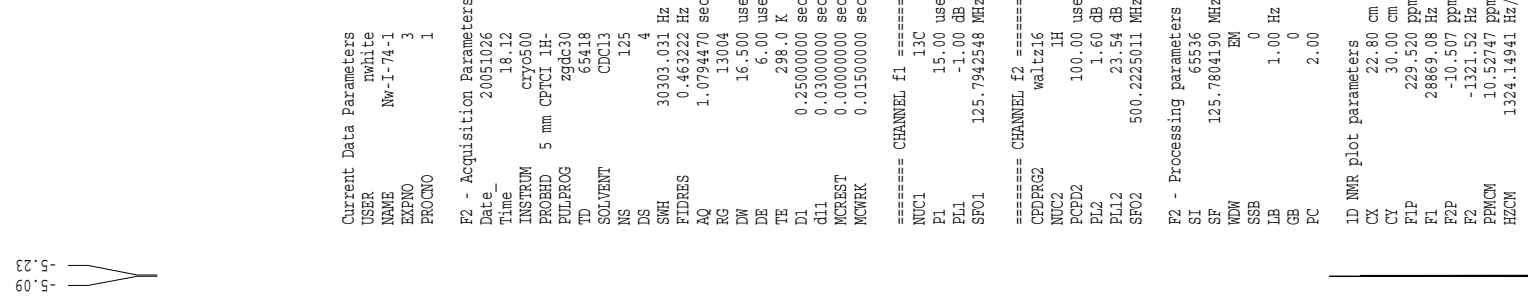

$\varepsilon \mp \cdot 8 \mathrm{I}$

$08 \cdot 57$
76.57

II.99

$28.9 L$
$\angle 0 . L L$

2E:LL

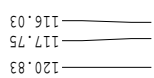

¿ะ'6Zโ

IT· `ร

OF. 891

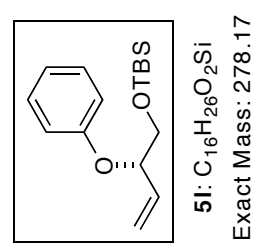




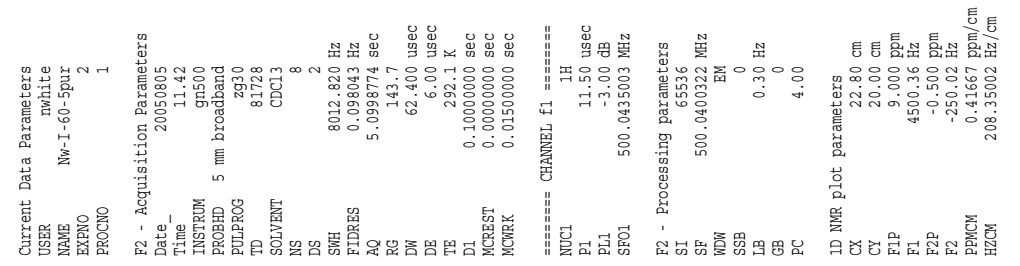

Kirsch, Overman, White

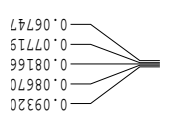

$29798^{\circ} \cdot 0$
$\angle 8 T \angle 8 \cdot 0$

¿ร\&68:0

ITOT6 ${ }^{\circ} 0$

86956.0-

$60426^{\circ} 0$

$28699^{\circ} \mathrm{T}$

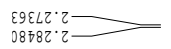

$\angle 80 Z L \cdot \varepsilon-$
$Z \angle 0 \varepsilon L \cdot \varepsilon-$

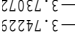

万IZSL

$26278^{\circ} \varepsilon$
$\angle T 9 \varepsilon 8^{\circ} \varepsilon$

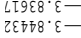

$\left\ulcorner\varepsilon \mp \mp 8^{\circ} \varepsilon-\right.$
$\sigma \varsigma \angle 98^{\circ} \varepsilon-$

OFSE

IZ8 $289^{\circ} \mathrm{A}$

$\angle 08 \varepsilon Z^{\circ} \mathrm{g}$

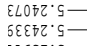

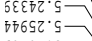

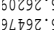

9ц8ट

9тtz\&: 9

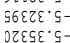

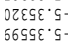

$0889 \varepsilon^{\circ} \mathrm{c}$

68988.

$92878^{\circ} \cdot \mathrm{c}$

$92898^{\circ} \mathrm{c}$

t)

ந9TL8. 5

60868.5

$86706 \div$
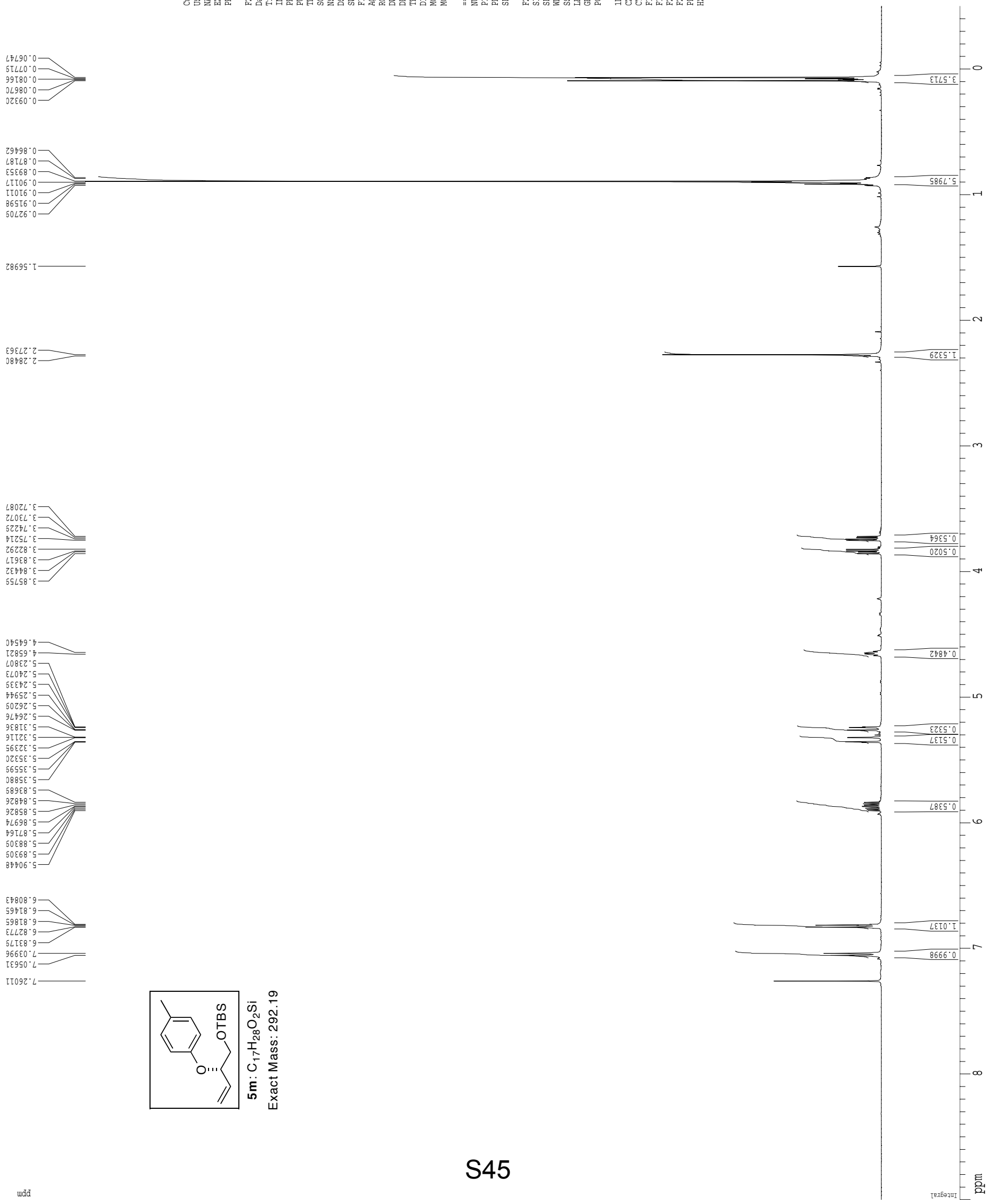
Kirsch, ${ }_{5}$ Overman, White
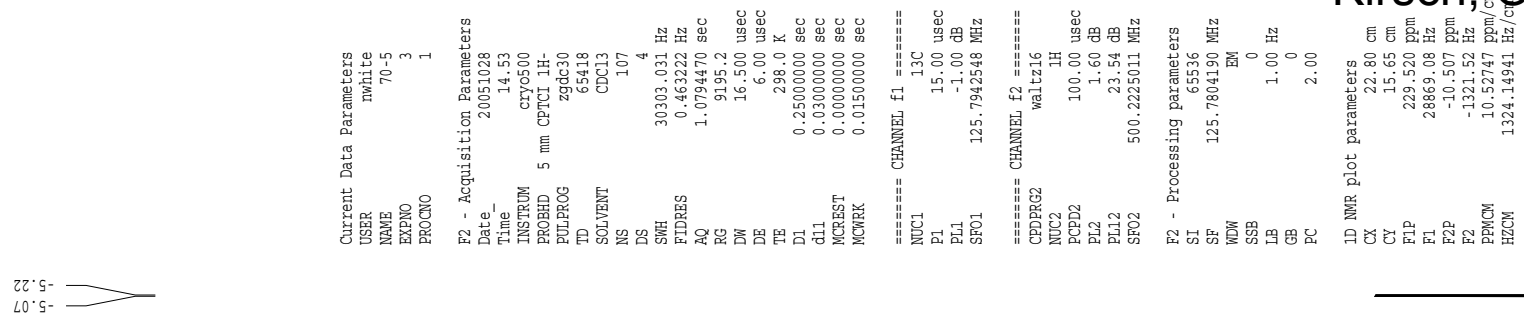

$\varepsilon \mp \cdot 8 \tau=$
$79.02 \square$
96.92
00.92

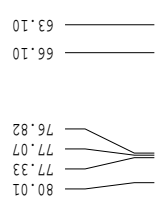

$\frac{59^{\circ} \mathrm{fIT}}{86^{\circ} \mathrm{SIT}}$

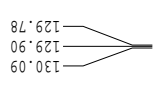

67.98

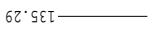

૬२.9९โ

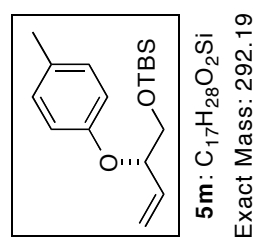




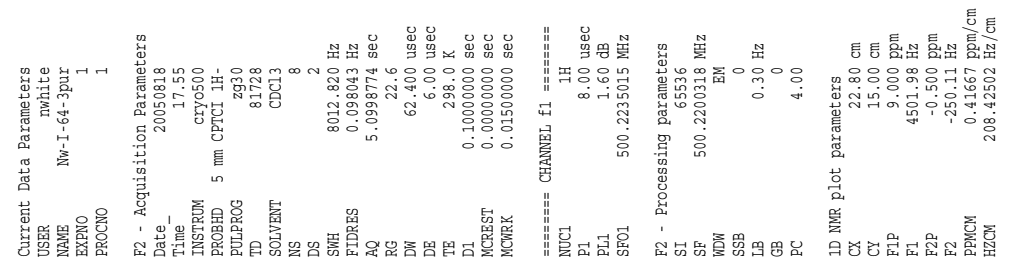

Kirsch, Overman, White

$25790^{\circ} 0$
$\varepsilon 7060^{\circ} 0$

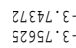

$529 S L^{\circ} \varepsilon$
$6 \varepsilon 99 L^{\circ} \varepsilon-$

$\angle 2608^{\circ} \varepsilon$
$08278^{\circ} \varepsilon-\varepsilon$

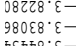

${ }^{86068} \cdot 0 \longrightarrow$

IIEF9:

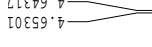

$87092 \cdot 5$

$76 \mathrm{t8} \cdot \mathrm{s}$

6LLFE'

$\varepsilon 6978.9$

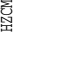


Kirsch, ${ }_{5}$ Overman, White

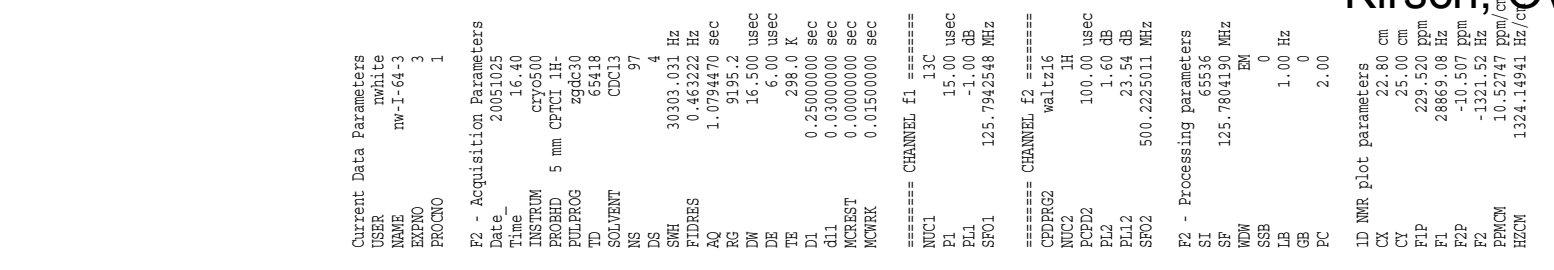

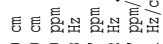

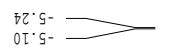
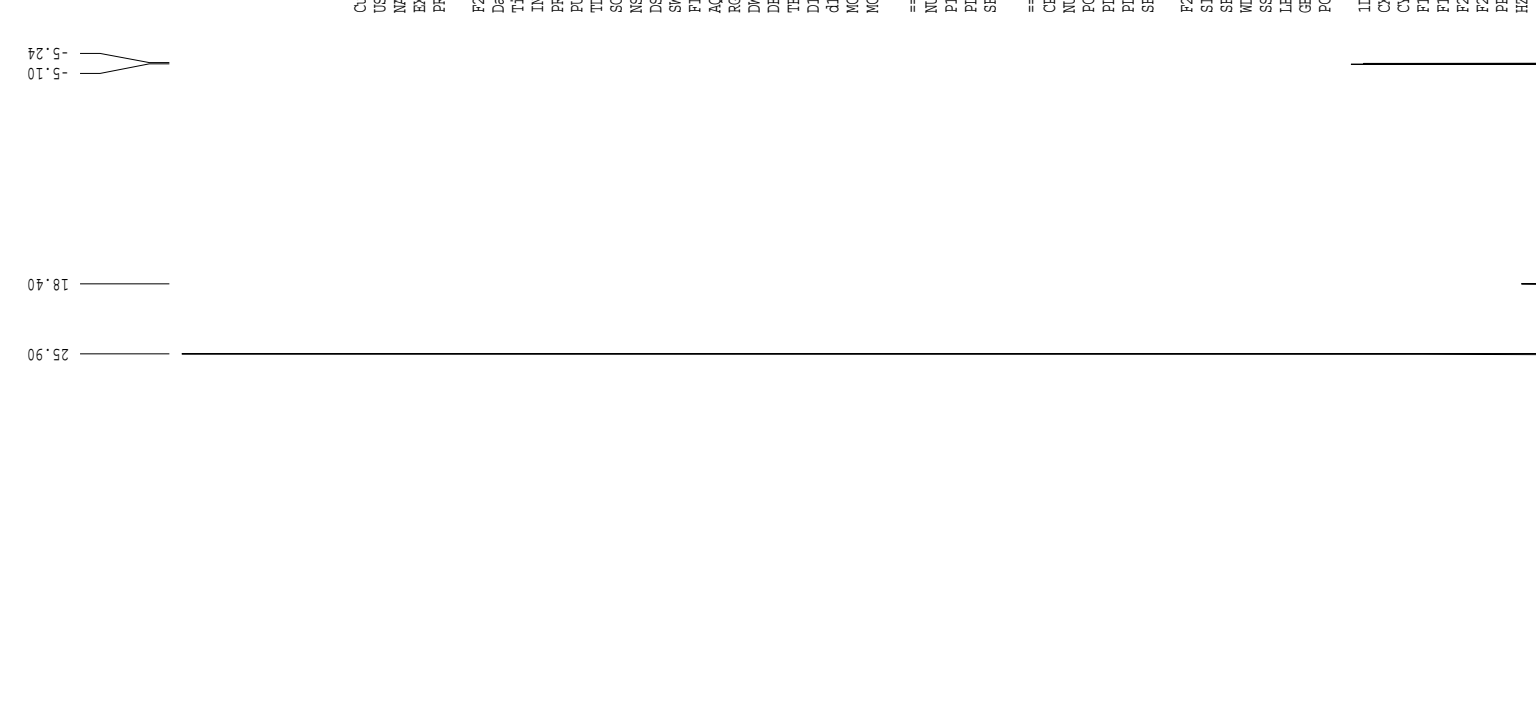

50.99

$78 \cdot 9 L$
$\angle 0 . \angle L$

$2 . L L$
$Z \nabla^{\circ} 08$

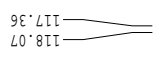

$\angle 9 \cdot 9 Z T-$
$0 Z \cdot 6 Z T$

$69^{\circ}$ ๆ

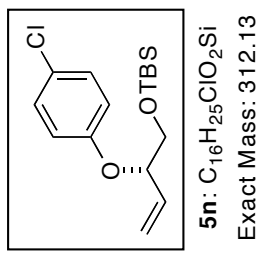

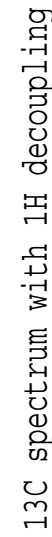




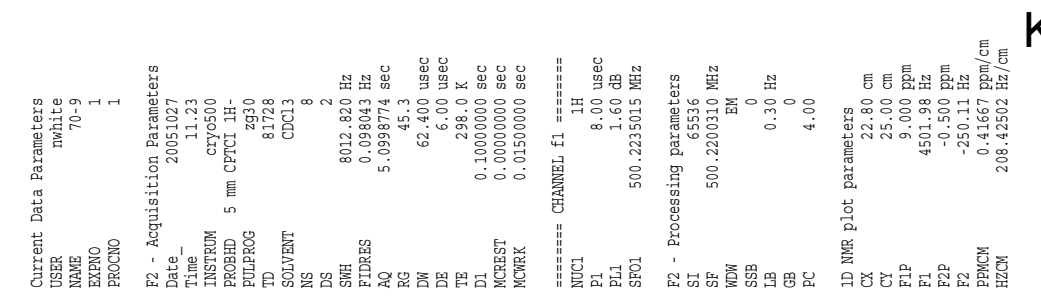

Kirsch, Overman, White
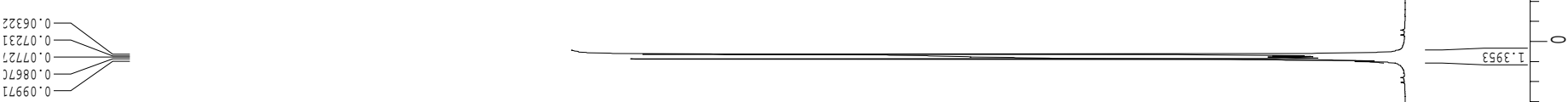

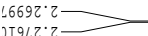

$5888 \cdot 0$

$27668^{\circ} \cdot 0$

$56606^{\circ} 0$

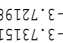

ESEтL' $\varepsilon=$

โО\&SL $\varepsilon$

$58078 \cdot \varepsilon$

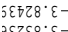

$\exists \varepsilon ₹ \varepsilon 8^{\circ} \varepsilon-$

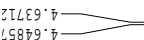

$26292 \cdot 9$

โย๊LL.

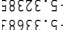

EL9ع 's

โ9728.

689978.9
68.9

$57 L 98^{\circ} 9$

$72768 \cdot 9$

$28968 \cdot 9$
$7016 \cdot 9$

)ร826.9-

$5 \angle 8 \varepsilon 6 \cdot 9$

गโร76.9-

$3 \angle 676 \cdot 9$

$3 \angle 896 \cdot 9$

$52 \varepsilon 96 \cdot 9$

$52896 \cdot 9=$
LT $092 \cdot \mathrm{L}$

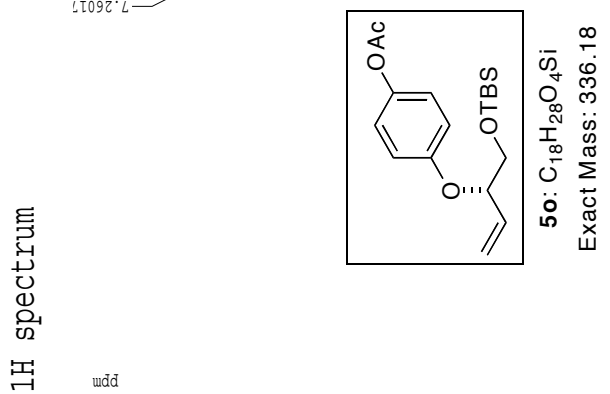

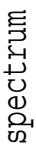


Kirsch, ${ }_{5}$ Overman, White
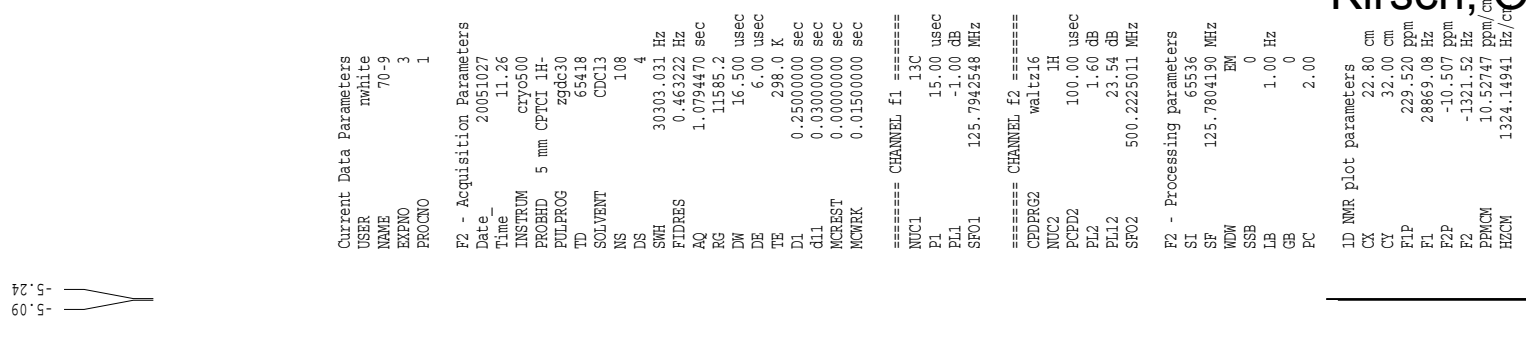

$27.8 \mathrm{~T}$

SI' $\tau$ I

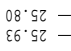

$6 \varepsilon .99$
80.99

$28.9 L$
$\angle 0 . \angle L$

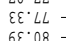

$6 \varepsilon^{\circ} 08$
$\angle F^{\circ} 08$

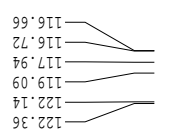

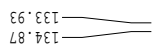

$82^{\circ} \mp \hbar \mathrm{L}$

EI.9G -
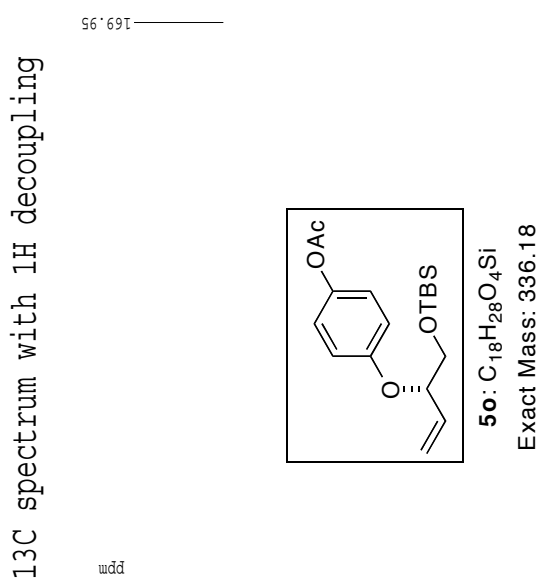


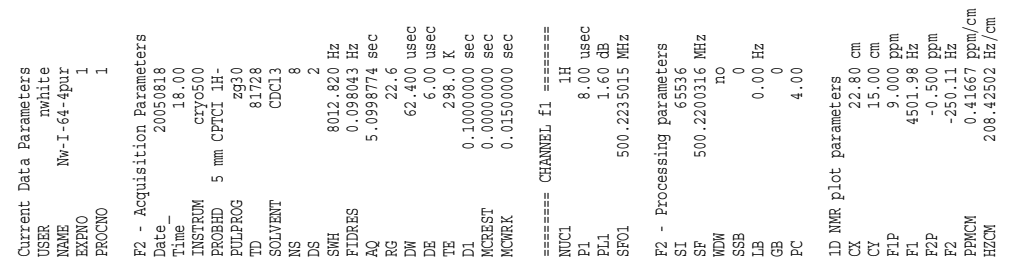

Kirsch, Overman, White

$\mp \angle 270.0-$

$76990^{\circ} 0$

$90 T \angle 0^{\circ} 0-$

EIEL8:0

$\angle \varepsilon 6 \angle 8^{\circ} \circ$

¿8288.0

E0968.0

$\doteqdot 7808^{\circ} \varepsilon-$

$\angle 8278^{\circ} \varepsilon-$

$\angle 6628^{\circ} \varepsilon$

$69788^{\circ} \varepsilon$
$596 \mathrm{~T} 6^{\circ} \varepsilon$

$596 \mathrm{~T} 6^{\circ} \varepsilon-$
$98 \mathrm{TE} \cdot \mathrm{C}$

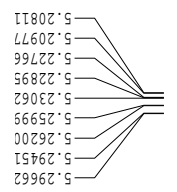

ธะระ8.9-

ธELE8.9-

$60298 \cdot 9$
$27898^{\circ} \cdot 9$

$8 \varepsilon 806^{\circ} \cdot$

†हाT6.

$50926 \cdot 9$

Oโ69Z ${ }^{\circ} L$
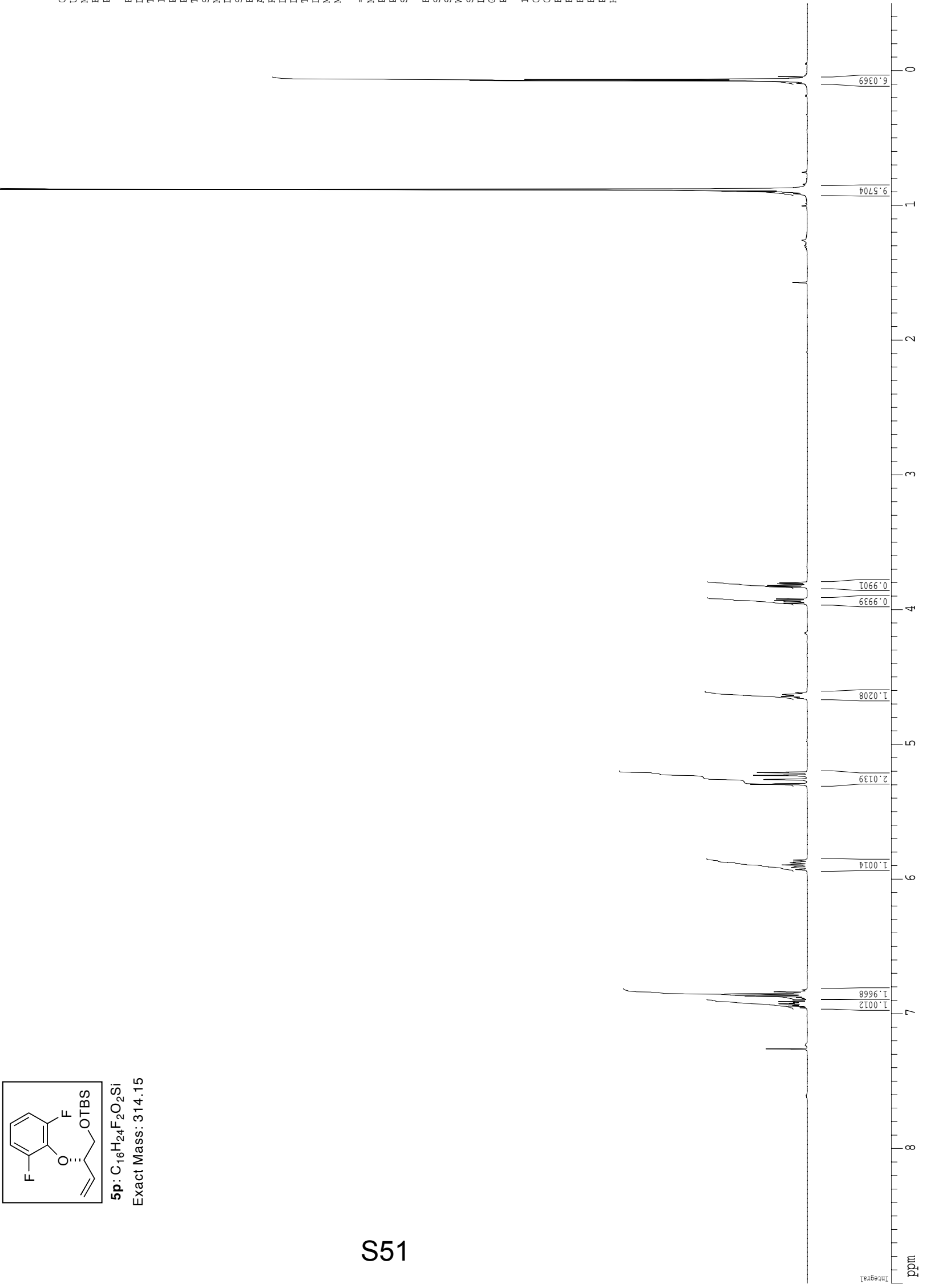
Kirsch, ${ }_{\text {OS }}$ Overman, White

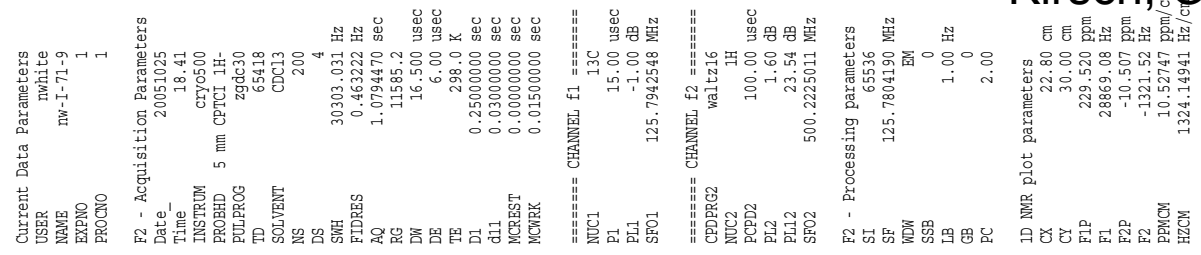

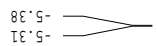

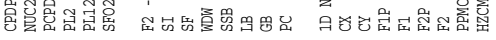

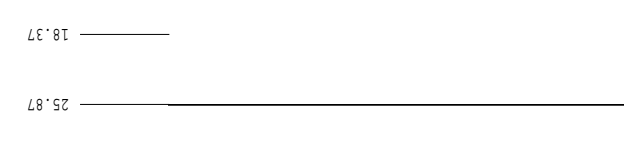

88.59

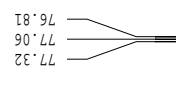

69.58

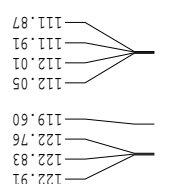

$\angle F^{\circ} \mp \varepsilon I-$

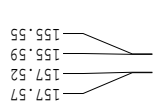

Z5. $\angle S T$

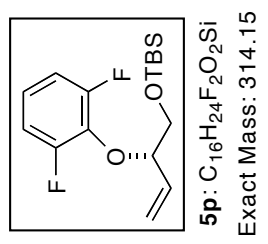




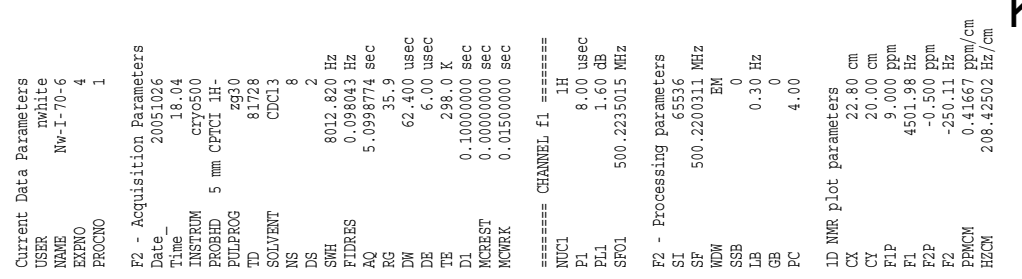

Kirsch, Overman, White
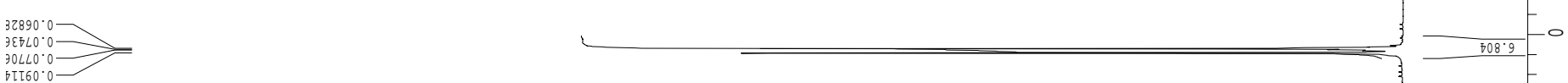

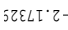

$6598 \cdot 0$

50268.0

$97668^{\circ} 0$

I.0ZT6.0

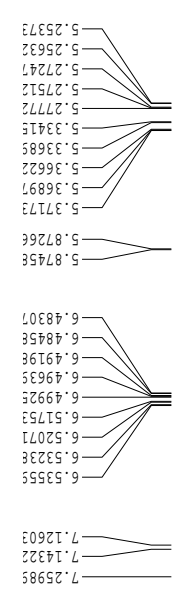

豆
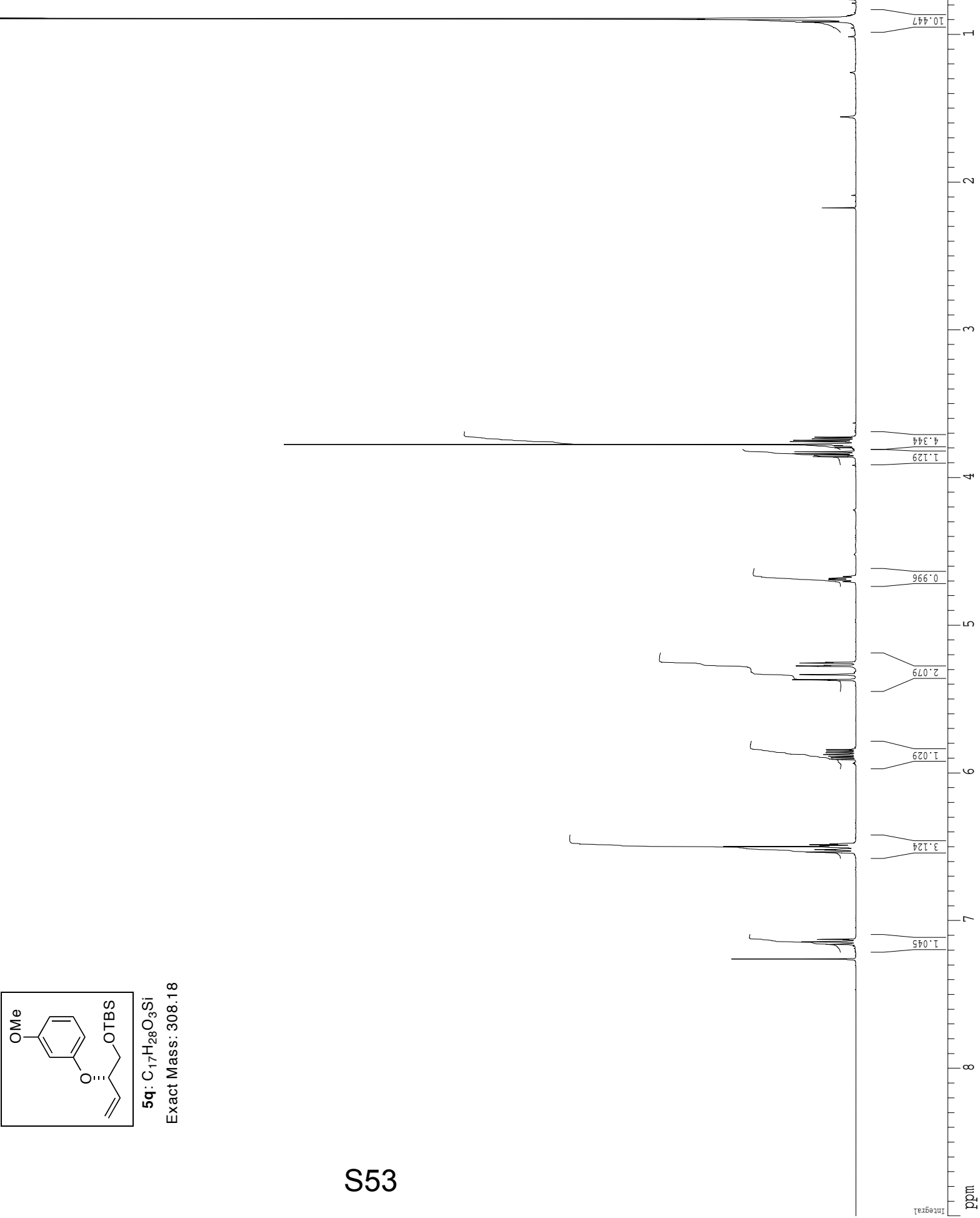
Kirsch, Everman, White
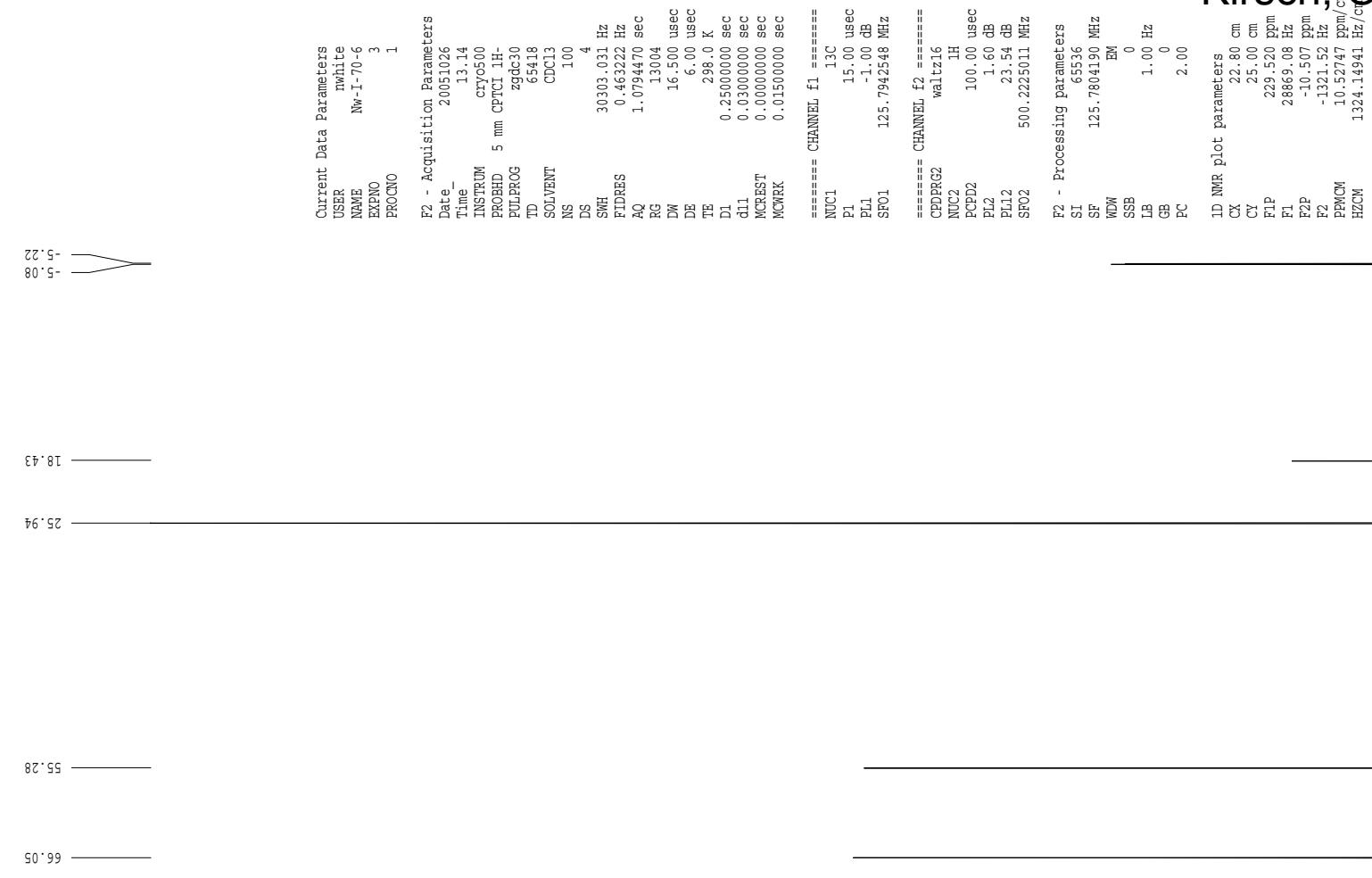

$28.9 L$
$L 0 . L L$

$\angle 0^{\circ} L L=$
$\varepsilon \varepsilon . L L=$
$58.6 L$

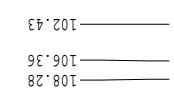

$8 L \cdot L I T$

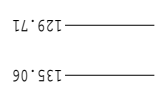

$79 \cdot 69 \tau-$
TL.09โ
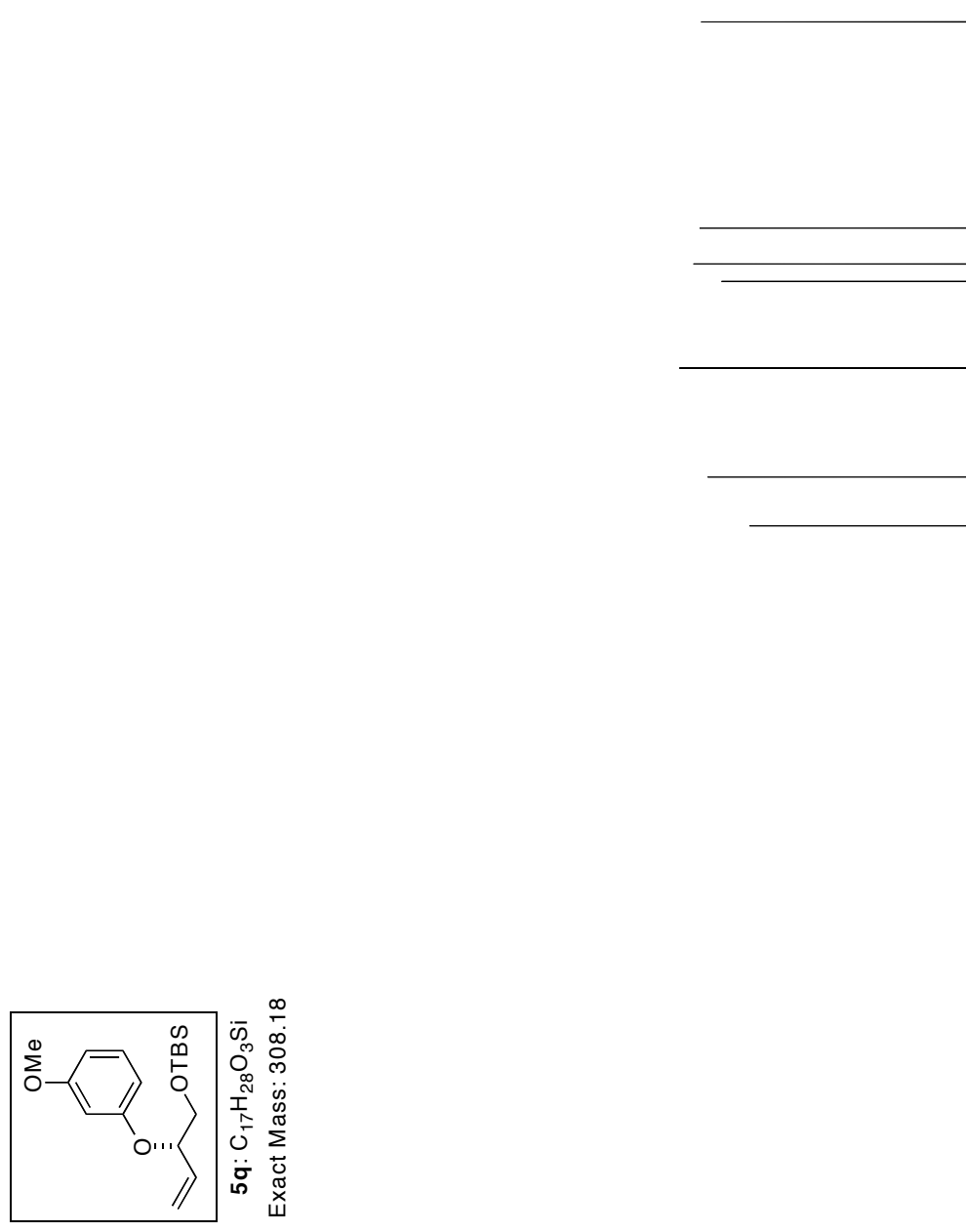